$\begin{array}{cc}\text { Programa de Pós-Graduação em Engenharia de Produção - PPGEP } & \\ \text { Laboratório de Qualidade de Vida - LaQVida } & \text { REVISTA BRASILEIRA DE } \\ \text { Universidade Tecnológica Federal do Paraná - UTFPR } & \text { Ponta Grossa - PR - Brasil } \\ \text { v. 07, n. 01, jan./mar. 2015, p. } 27-38 & \text { QUALIDADE DE VIDA }\end{array}$

\title{
Qualidade de vida relacionada à saúde em servidores públicos
}

\section{Civil servants' quality of life related to the Health}

\author{
Andreia Hirt Santos \\ Universidade Estadual do Oeste do Paraná - UNIOESTE - Cascavel - Paraná - Brasil \\ andreia_hirt@hotmail.com \\ Karen Josiane Soares Pereira \\ Universidade Estadual do Oeste do Paraná - UNIOESTE - Cascavel - Paraná - Brasil \\ karenjspereira@gmail.com \\ Claudia Ross \\ Universidade Estadual do Oeste do Paraná - UNIOESTE - Cascavel - Paraná - Brasil \\ claudiross@gmail.com \\ Ligiane de Lourdes da Silva \\ Universidade Estadual do Oeste do Paraná - UNIOESTE - Cascavel - Paraná - Brasil \\ ligianes@gmail.com
}

\section{RESUMO}

OBJETIVO: Avaliar a qualidade de vida relacionada à saúde (QVRS) em servidores públicos estaduais de uma instituição de ensino superior do oeste do Paraná-Brasil.

MÉTODOS: Trata-se de um estudo descritivo, correlacional e transversal realizado por meio do instrumento Medical Outcomes Study (SF-36) para a avaliação da QVRS. As variáveis foram analisadas pelo programa Statistical Package for the Social Sciencies (SPSS) versão 15.0 e aplicado o teste t de Student e teste de Tukey. Para a rejeição da hipótese de nulidade foi considerado o nível de significância $\mathrm{p}<0,05$.

RESULTADOS: Participaram da pesquisa 110 indivíduos, sendo a maioria do sexo feminino (70,0\%), faixa etária de 41 a 60 anos (51,8\%) e 59,1\% casados. Quanto à escolaridade, 56,4\% da amostra possui mais que oito anos de estudo formal. As médias encontradas dos domínios foram: Capacidade funcional $(83,45)$, Limitações por aspectos físicos $(84,32)$, Limitações por aspectos emocionais $(86,06)$, e Aspectos sociais $(78,98)$ apresentaram os escores mais elevados. Enquanto Dor $(64,33)$, Estado geral de saúde $(71,81)$, Saúde mental $(70,33)$ e Vitalidade $(65,86)$ demonstraram-se mais comprometidos na população estudada.

CONCLUSÕES: O SF-36 demonstrou-se um instrumento muito útil no rastreamento de necessidades da QVRS, evidenciando maior acometimento da saúde para os domínios Dor e Vitalidade.

PALAVRAS-CHAVE: Qualidade de vida. Saúde do trabalhador. Servidor público. 


\section{ABSTRACT}

OBJECTIVE: Evaluating the quality of life related to health (HRQoL) in state civil servants of a higher education institution in Western Paraná, Brazil.

METHODS: It was a descriptive, correlational and cross-sectional study conducted by the Medical Outcomes Study instrument (SF-36) to assess HRQoL. The variables were analyzed using the Statistical Package for the Social Sciences (SPSS) version 15.0 and the test t was applied of Student and also the Tukey test. To reject the null hypothesis the significance level of $p<0.05$ was considered. RESULTS: One hundred and ten individuals took part in the trial, mostly female (70\%), aged 41-60 years $(51.8 \%)$ and $59.1 \%$ were married. As for education, $56.4 \%$ of the individuals have more than eight years of formal education. The average percentages of dominance were: Functional capacity (83.45), Limitations for physical aspects (84.32), Limitations for emotional aspects (86.06) and for Social aspects (78.98) those were the higher scores. While pain (64.33), General health (71.81), Mental health (70.33) and Vitality (65.86) were far more harmed in the study population.

CONCLUSIONS: The SF-36 proved to be a very useful tool in tracking HRQoL needs, showing greater involvement of health for the dominance of Pain and Vitality.

KEYWORDS: Quality of life. Occupational health. Public servant.

\section{Introdução}

A qualidade de vida (QV) é uma concepção eminentemente humana, uma construção individual que tenta expressar o grau de contentamento encontrado na vida familiar, social, ambiental, amorosa, envolvendo para tanto o próprio conceito de existência humana (URZUA, 2010).

O termo QV é muito amplo, e se aplica de diferentes formas conforme o interlocutor, o local e a época. Suas distintas vertentes estão distribuídas em múltiplas áreas de estudo, apresentando-se como QV ligada à política, à economia, à ergonomia, relacionada à saúde e ao trabalho. Ainda que subjetivo, o estudo da QV é importante para tomada de decisões clínicas, como avaliação de programas de acompanhamento de saúde, alocação de recursos e aprovação de diferentes intervenções (BERLIM; FLECK, 2003).

No contexto médico, emprega-se o termo como aspectos referentes à experiência individuais, relacionados à saúde e aos cuidados em saúde. Logo, busca-se um sinônimo da QV que exprima mais precisamente o termo médico proposto, apresentando então a Qualidade de Vida Relacionada à Saúde (QVRS).

Não se pode separar a doença da perspectiva social e pessoal do indivíduo. Desse modo, para entender melhor o que afeta a vida e saúde do sujeito se torna essencial capturar suas perspectivas particulares em diferentes situações clínicas (BERLIM; FLECK, 2003, SCHULZ et al., 2008). Assim, percebe-se a QVRS como uma noção extremamente individual, onde a valorização da opinião do sujeito é o primeiro passo em busca da compreensão dos fatores que, de alguma forma, afetam a QV ligada a saúde do mesmo.

A medida da QVRS fornece uma base de dados úteis para o monitoramento dos efeitos e progressão de uma doença, assim como seu tratamento, sendo útil tanto para o paciente, quanto para o médico (FARHAT; ZAZINI, 1998).

O uso dos dados gerados pelo estudo da QVRS, quando realizado em uma população homogênea, pode ser útil para o rastreamento de necessidades de saúde nestes indivíduos. Parte-se, então, para o pressuposto de que, identificadas às necessidades de um grupo populacional, é possível o desenvolvimentos de estratégias de promoção de saúde para os sujeitos de forma concisa e objetiva.

Para quantificar a QVRS é imprescindível que sejam realizados estudos relacionados sobre a doença, seus tratamentos e como ambos afetam a duração da vida e a qualidade da mesma. Para tal são utilizados instrumentos de pesquisa, os quais tentam transcrever as variáveis mensuráveis e passíveis de emprego por meio de comparações entre as diferentes intervenções (FARHAT; ZAZINI, 1998). 
A utilização de instrumentos validados é de grande valia, já que pode ser utilizada para estudos multicêntricos e internacionais, possibilitando comparações com diferentes populações, sem requisitar dispendiosos investimentos (SCHULZ et al., 2008).

Neste estudo foi utilizado um instrumento genérico, traduzido e validado, o Medical Outcomes Study Short-Form 36 (SF-36). Este questionário apresenta facilidade de aplicação, brevidade e grande abrangência, uma vez que possui perguntas curtas e questões múltiplas. Possui 36 questões divididas em dois componentes de saúde: Componente saúde física (CSF) e Componente saúde mental (CSM). Estes componentes subdividem-se em oito domínios, sendo eles, para CSF: Capacidade funcional, Limitação por aspectos físicos e Dor, para CSM: Limitações por aspectos emocionais, Saúde mental e Aspectos sociais, e os domínios Vitalidade e Estado geral de saúde relacionados a ambos os componentes. A escala utilizada é de zero a 100, sendo zero estado de saúde menos favorável, consequentemente a pior QV, e 100 o melhor estado de saúde e consequentemente a melhor QV (CICONELLI et al., 1999; MARTINS; CESARINO, 2005; WARE JR., 2000).

Para algumas doenças, o trabalho é considerado como um fator contributivo, que colabora de forma inespecífica para a morbidade ou agravo de doenças. Mendes (1988) relata o estresse, a carga horária, a organização do trabalho, o trabalho em turnos, e a sobrecarga psíquica como sendo riscos atribuídos à degradação da saúde do trabalhador.

É necessário que os danos à saúde causados pelo trabalho sejam observados atentamente por profissionais da assistência médica. Deve-se buscar métodos de rastreamento de pontos críticos relacionado ao tipo de trabalho, que interfiram diretamente na QVRS. Esse grupo de atitudes visa entender as necessidades dos diferentes tipos de trabalhadores, e esquadrinhar meios de interceptação dos danos, levando à prevenção de futuros agravos à saúde do sujeito.

O objetivo deste estudo foi avaliar a QVRS em servidores públicos estaduais de uma instituição de ensino superior do oeste do Paraná-Brasil.

\section{Metodologia}

Trata-se de um estudo descritivo, correlacional e transversal. A população estudada foi determinada a partir de uma lista disponibilizada pelo setor de recursos humanos de uma universidade pública da cidade de Cascavel, Paraná, Brasil. Os critérios de inclusão foram os servidores públicos da instituição alocados no campus de Cascavel; e, de exclusão, os servidores em férias, licenças, cedidos para outros campi, funcionários terceirizados ou aqueles que se negaram a participar da pesquisa e a assinar o Termo de Consentimento Livre e Esclarecido.

A coleta de dados foi realizada no período de dezembro de 2011 a janeiro de 2012, por meio de entrevistas individuais realizadas pelas pesquisadoras e por acadêmicos do curso de Farmácia previamente treinados e capacitados. O projeto de pesquisa foi apresentado de modo verbal a todos os participantes da pesquisa e, após entendimento e consentimento destes, foi assinado o Termo de Compromisso Livre e Esclarecido, aprovado pelo Comitê de Ética em Pesquisa (CEP), sob parecer $n^{\circ}$ 457/2011-CEP.

O questionário geral de QVRS utilizado foi o Medical Outcomes Study Short-Form (SF-36) composto de 36 perguntas, subdividido em 8 domínios (Capacidade funcional, Dor, Estado geral de saúde, Vitalidade, Aspectos físicos, Aspectos emocionais, Saúde mental e Aspectos sociais) traduzido e validado por Ciconelli et al.(1999).

Para a descrição do perfil dos servidores foi elaborado um instrumento com dados sociodemográficos e socioeconômicos: sexo, idade, estado civil, grau de escolaridade, renda familiar, tempo de serviço na instituição e turno de trabalho, e dados sobre as condições de saúde: altura, peso e enfermidades autorreferidas.

O índice de massa corporal (IMC) foi calculado pelo peso $(\mathrm{kg})$ dividido pela altura ao quadrado. Os resultados foram classificados conforme recomendações da World Health Organization (2008), sendo classificados em: Desnutrição abaixo de $18,49 \mathrm{Kg} / \mathrm{m}^{2}$; Faixa de Peso Normal entre $18,50 \mathrm{Kg} / \mathrm{m}^{2}$ e $24,99 \mathrm{Kg} / \mathrm{m}^{2}$; Sobrepeso entre $25 \mathrm{Kg} / \mathrm{m}^{2}$ e $29,99 \mathrm{Kg} / \mathrm{m}^{2}$; e Obesidade acima $30 \mathrm{Kg} / \mathrm{m}^{2}$.

Os dados foram tabelados em uma planilha do programa Microsoft Office Excel® 2009, 
posteriormente transferidos para o programa Statistical Package for the Social Sciencies (SPSS), versão 15.0, no qual foram processados e avaliados. Foi realizada análise exploratória para reconhecimento das variáveis e correção de eventuais erros e/ou inconsistências na coleta e na digitação dos dados.

A análise dos dados constituiu-se na descrição das frequências absolutas e relativas na realização de testes de associação para as variáveis: classificação da escolaridade e nível de QV. As variáveis contínuas foram apresentadas na forma de média de desvio padrão e analisadas pelo teste $\mathrm{t}$ de Student $\mathrm{e}$ teste de Tukey. Para a rejeição da hipótese de nulidade foi considerado o nível de 0,05 (ou 5,0\%).

\section{Resultados e discussão}

\subsection{Perfil sociodemográfico e socioeconômico}

Do total de 155 servidores públicos, 117 foram elegíveis para a participação do estudo e 110 aceitaram participar e completaram a entrevista para compor a amostra do estudo. As características sociodemográficas e socioeconômicas dos servidores pesquisadas estão descritas na Tabela 1:

Tabela 1 - Características sociodemográficas e socioeconômicas de servidores de uma universidade pública da cidade de Cascavel

\begin{tabular}{|c|c|c|c|}
\hline Variável & Classes & $\mathbf{N}$ & Porcentagem \\
\hline \multirow{2}{*}{ Sexo } & Feminino & 77 & $70,0 \%$ \\
\hline & Masculino & 33 & $30,0 \%$ \\
\hline \multirow[t]{3}{*}{ Faixa etária } & Até 40 anos & 42 & $38,2 \%$ \\
\hline & De 41 a 60 anos & 57 & $51,8 \%$ \\
\hline & Mais de 60 & 11 & $10,0 \%$ \\
\hline \multirow[t]{5}{*}{ Estado Civil } & Solteiro(a) & 28 & $25,5 \%$ \\
\hline & União estável & 1 & $0,9 \%$ \\
\hline & Casado(a) & 65 & $59,1 \%$ \\
\hline & Separado(a) & 14 & $12,7 \%$ \\
\hline & Viúvo(a) & 2 & $1,8 \%$ \\
\hline \multirow[t]{3}{*}{ Escolaridade } & 1 a 4 anos de estudo & 6 & $5,5 \%$ \\
\hline & 5 a 8 anos de estudo & 39 & $35,5 \%$ \\
\hline & Acima de 8 anos de estudo & 65 & $59,0 \%$ \\
\hline \multirow[t]{3}{*}{ Renda } & Não foi respondido & 1 & $0,9 \%$ \\
\hline & 1 a 5 salários mínimos & 62 & $56,4 \%$ \\
\hline & Mais de 5 salários mínimos & 47 & $42,7 \%$ \\
\hline \multirow[t]{7}{*}{ Tempo de serviço } & Menos de 5 anos & 5 & $4,5 \%$ \\
\hline & De 6 a 10 anos & 32 & $29,1 \%$ \\
\hline & De 11 a 15 anos & 15 & $13,6 \%$ \\
\hline & De 16 a 20 anos & 34 & $30,9 \%$ \\
\hline & De 21 a 25 anos & 19 & $17,3 \%$ \\
\hline & De 26 a 30 anos & 4 & $3,6 \%$ \\
\hline & Mais de 30 anos & 1 & $1,0 \%$ \\
\hline
\end{tabular}

Verificou-se a predominância do sexo feminino, o que corrobora com outro estudo realizado com servidores públicos (PINHEIRO; SUGAHARA, 2001). A partir da década de 70 houve a progressão do ingresso da mulher no mercado de trabalho, influenciada pela redução da taxa de fecundidade e o aumento do nível de instrução da população feminina, abrindo espaço para maiores perspectivas no ambiente de trabalho (PROBST; RAMOS, 2003).

A idade variou de 25 a 69 anos, com prevalência da faixa etária entre 41 a 60 anos $(51,8 \%)$ (Tabela 1), o mesmo foi observado por Pinheiro e Sugahara (2001). Quanto ao estado civil, 59,1\% dos indivíduos são casados. No que se refere à renda mensal e escolaridade, 56,4\% apresenta renda mensal 
de um a cinco salários mínimos e 59,0\% possuem mais de oito anos de estudo formal.

Em relação aos cargos ocupados, os mais prevalentes foram: Técnico Administrativo (31,8\%), seguido de Técnico de Laboratório $(16,4 \%)$ e Auxiliar Operacional $(13,6 \%)$, o restante se distribuiu em diversas outras funções.

\subsection{Qualidade de vida relacionada à saúde (QVRS)} Tabela 2 .

Os escores médios das dimensões obtidas com a aplicação do SF-36 estão apresentados na

Tabela 2 - Aspectos gerais da Qualidade de Vida de servidores de uma universidade pública da cidade de Cascavel

\begin{tabular}{lcccc}
\hline \multirow{2}{*}{ DOMíNIOS } & Média & DP & \multicolumn{3}{c}{ Intervalo de conf. 95\% } \\
\cline { 4 - 5 } & & & LI & LS \\
\hline Capacidade funcional & 83,45 & 15,23 & 81,05 & 85,86 \\
Limitação por aspectos físicos & 84,32 & 26,85 & 80,08 & 88,55 \\
Dor & 64,33 & 20,36 & 61,12 & 67,54 \\
Estado geral de saúde & 71,81 & 17,47 & 69,06 & 74,56 \\
Vitalidade & 65,86 & 18,75 & 62,91 & 68,82 \\
Aspectos sociais & 78,98 & 21,61 & 75,57 & 82,38 \\
Limitações por aspectos emocionais & 86,06 & 28,33 & 81,59 & 90,53 \\
Saúde mental & 70,33 & 18,74 & 67,37 & 73,28 \\
\hline
\end{tabular}

Obs: LI: Limite inferior; LS: Limite superior.

Fonte: Autoria própria (2012).

$\mathrm{Na}$ análise geral da QV os resultados demonstram que para a população estudada os valores médios de QVRS foram elevados, ou seja, acima de 70,0 para a maioria dos domínios, revelando pouco comprometimento na saúde do grupo. Contudo, é importante também conhecer os resultados de cada domínio, verificar os mais afetados e compreender as causas de prejuízo para QVRS.

De acordo com a Tabela 2, os domínios mais comprometidos foram Dor $(64,33 \pm 20,36)$ e Vitalidade $(65,86 \pm 18,75)$, o que indica a presença de dor extrema ou limitante. Tal situação pode ser decorrente de condições autorreferidas pelos servidores como o distúrbio osteomuscular relacionado ao trabalho (DORT). Já para o domínio Vitalidade, a diminuição da disposição e o ânimo de enfrentar dificuldades do dia-a-dia são causas possíveis do resultado. Este domínio também é altamente sensível a algumas patologias como hipertensão arterial e depressão, além de estar relacionado à fadiga ligada à idade e ao tempo de doença (WARE JR.; SHERBOURNE, 1992; SILQUEIRA, 2005; CARVALHO et al., 2012).

Para os outros domínios as pontuações foram acima de 70,0. O maior escore foi demonstrado para o domínio Limitações por aspectos emocionais $(86,06 \pm 28,33)$, o qual se relaciona com as interferências dos problemas emocionais nas atividades do dia-a-dia e no trabalho, seguido do domínio Limitações por aspectos físicos $(84,32 \pm 26,85)$, onde é refletida a ausência de problemas físicos que resultem em dificuldades no trabalho ou em execução de atividades diárias.

Para o domínio Capacidade funcional $(83,45 \pm 15,23)$, observou-se alta pontuação na população estudada demonstrando uma boa condição física, também verificada no domínio Aspecto físico. Estes dois domínios demonstram que os servidores apresentam pouca limitação quanto ao tipo de trabalho na atividade de vida diária.

O resultado encontrado para o domínio Aspectos sociais $(78,99 \pm 21,61)$ permite inferir que as relações sociais ou atividades de lazer são raramente afetadas por problemas físicos e/ou emocionais. Para o domínio Estado geral de saúde $(71,81 \pm 17,47)$ verificou-se que os servidores têm uma boa percepção de saúde, bem como são capazes de detectar a melhora ou piora da mesma com o passar do tempo.

Já para o domínio Saúde mental $(70,33 \pm 18,74)$, o valor encontrado possui um maior grau de comprometimento em relação aos outros, porém, não é considerado baixo, demonstrando que os servidores têm um bom equilíbrio emocional e que, quando encontrados valores inferiores, estes estão 
correlacionados a sensações de depressão e de nervosismo (WARE JR.; SHERBOURNE, 1992).

A Tabela 3 apresenta os resultados dos valores dos domínios do SF-36 de acordo com os diferentes grupos etários, calculado a partir da média aritmética, desvio padrão e teste de significância $(\mathrm{p}<0,05)$.

Tabela 3 - Correlação entre os domínios do SF-36 com as faixas etárias dos servidores de uma universidade pública na cidade de Cascavel

\begin{tabular}{|c|c|c|c|c|}
\hline & Classificação da idade & Média & Desvio padrão & p-valor \\
\hline \multirow[t]{3}{*}{ Capacidade funcional } & Até 40 anos & 85,48 & 15,13 & 0,483 \\
\hline & De 41 a 60 anos & 82,63 & 15,50 & \\
\hline & Mais que 60 anos & 80,00 & 14,49 & \\
\hline \multirow[t]{3}{*}{ Limitação por aspectos físicos } & Até 40 anos & 78,57 & 32,47 & 0,186 \\
\hline & De 41 a 60 anos & 88,60 & 21,69 & \\
\hline & Mais que 60 anos & 84,09 & 25,67 & \\
\hline \multirow[t]{3}{*}{ Dor } & Até 40 anos & 65,00 & 18,05 & 0,933 \\
\hline & De 41 a 60 anos & 63,63 & 22,22 & \\
\hline & Mais que 60 anos & 65,36 & 20,37 & \\
\hline \multirow[t]{3}{*}{ Estado geral de saúde } & Até 40 anos & 73,60 & 18,71 & 0,691 \\
\hline & De 41 a 60 anos & 70,89 & 15,65 & \\
\hline & Mais que 60 anos & 69,73 & 22,29 & \\
\hline \multirow[t]{3}{*}{ Vitalidade } & Até 40 anos & 61,55 & 19,24 & $0,044 *$ \\
\hline & De 41 a 60 anos & 66,93 & 18,07 & \\
\hline & Mais que 60 anos & 76,82 & 16,47 & \\
\hline \multirow[t]{3}{*}{ Aspectos sociais } & Até 40 anos & 76,79 & 22,36 & 0,644 \\
\hline & De 41 a 60 anos & 79,82 & 22,25 & \\
\hline & Mais que 60 anos & 82,95 & 15,08 & \\
\hline \multirow[t]{3}{*}{ Limitações por aspectos emocionais } & Até 40 anos & 83,33 & 33,94 & 0,677 \\
\hline & De 41 a 60 anos & 87,13 & 25,78 & \\
\hline & Mais que 60 anos & 90,91 & 15,57 & \\
\hline \multirow[t]{3}{*}{ Saúde mental } & Até 40 anos & 66,95 & 21,84 & 0,332 \\
\hline & De 41 a 60 anos & 72,56 & 15,67 & \\
\hline & Mais que 60 anos & 71,64 & 20,51 & \\
\hline
\end{tabular}

Obs: *Análise de variância com teste de Tukey ao nível de 5,0\% de significância. Fonte: Autoria própria (2012).

O domínio Vitalidade foi o único resultado que apresentou relevância significativa $(\mathrm{p}<0,05)$ conforme o teste de Tukey quando relacionado à faixa etária. A faixa etária dos servidores com mais que 60 anos apresentou maiores pontuações quando comparada com as demais. Resultados semelhantes foram observados por Pimenta et al. (2008). Para Barreto (2005), resultados melhores de QVRS podem estar associados à capacidade de adaptação do ser humano. Ao longo da vida, o ser humano passa por diferentes adversidades e, sucessivamente, adapta-se a estas mudanças, o que leva a alteração da percepção das dificuldades vividas. Percebe-se, então, que indivíduos com mais idade tendem a tolerar mais facilmente contratempos do dia-a-dia que indivíduos mais jovens.

Em contrapartida, observou-se que, com o aumento da idade, dois domínios obtiveram escores menores do que para os indivíduos mais jovens, Capacidade funcional e Estado geral de saúde. Mesmo estes servidores com mais idade, apresentando conhecimento e maturidade, é importante considerar que com o avançar da idade surgem os problemas físicos, as doenças crônicas, o enfraquecimento, a perda de massa muscular entre outras patologias concernentes ao envelhecimento. Estes adventos podem ser correlacionados com a redução da Capacidade funcional e Estado geral de saúde, na medida em que ambos os domínios estão associados à saúde física do indivíduo (WARE JR.; SHERBOURNE, 1992; SILQUEIRA, 2005; CARVALHO et al., 2012).

Em relação ao perfil de saúde dos servidores, as condições crônicas autorreferidas mais frequentes foram: Obesidade com 26,40\% ( $n=29)$, Gastrite com 24,50\% ( $n=29)$, Doença osteomuscular relacionada com o trabalho (DORT) com 20,90\% ( $\mathrm{n}=23)$, Doenças respiratórias com 19,10\% $(\mathrm{n}=21)$, Depressão e Hipertensão arterial ambas com 17,30\% (n=19) e Artrite reumatoide com 6,4\% (n=7), 
podendo o servidor apresentar uma ou mais enfermidades.

A condição crônica de saúde provoca mudanças no cotidiano do indivíduo, sobrecarga física, emocional e financeira, desencadeando incertezas, dilemas e onerosos gastos, que por sua vez pode afetar a QV (ZORTEA, 2010). A doença crônica dificulta a inserção no processo produtivo e, consequente, acesso aos bens de consumo, além de desencadear no indivíduo alterações na capacidade física, autoestima, imagem corporal e relações com outras pessoas (MARTINS; FRANÇA; KIMURA, 1996). Neste sentido, conhecer as doenças crônicas e QVRS dos servidores tem importância para implantação de ações voltadas à melhoria das condições de vida, trabalho e saúde, criando a percepção de bem estar.

A Tabela 4 mostra a correlação dos domínios do SF-36 com as condições crônicas de saúde autorreferidas pelos servidores. As enfermidades mais frequentes nos servidores foram obesidade e gastrite.

Tabela 4 - Correlação entre os domínios do questionário SF-36 com sexo e condições crônicas de saúde autorreferidas em servidores de uma universidade pública, na cidade de Cascavel

(continuação)

\begin{tabular}{|c|c|c|c|c|c|c|c|c|c|c|}
\hline \multicolumn{3}{|l|}{ Domínios } & \multirow{2}{*}{$\begin{array}{c}\text { CF } \\
77\end{array}$} & \multirow{2}{*}{$\begin{array}{l}\text { LAF } \\
77\end{array}$} & \multirow{2}{*}{$\begin{array}{l}\text { Dor } \\
77\end{array}$} & \multirow{2}{*}{$\begin{array}{l}\text { EGS } \\
77\end{array}$} & \multirow{2}{*}{$\begin{array}{l}\text { Vit. } \\
77\end{array}$} & \multirow{2}{*}{$\begin{array}{c}\mathbf{A S} \\
77\end{array}$} & \multirow{2}{*}{$\begin{array}{l}\text { LAE } \\
77\end{array}$} & \multirow{2}{*}{$\begin{array}{c}\text { SM } \\
77\end{array}$} \\
\hline \multirow{7}{*}{ Sexo } & $\mathrm{F}$ & $\mathrm{N}$ & & & & & & & & \\
\hline & & Média & 80,9 & 80,8 & 59 & 69,7 & 61,8 & 75,3 & 82,2 & 66,3 \\
\hline & & $\mathrm{Dp}$ & 15,8 & 30 & 19,4 & 17,8 & 18,5 & 23,3 & 30,8 & 18,6 \\
\hline & $\mathrm{M}$ & $\mathrm{N}$ & 33 & 33 & 33 & 33 & 33 & 33 & 33 & 33 \\
\hline & & Média & 89,4 & 92,4 & 76,7 & 76,7 & 75,3 & 87,5 & 94,9 & 79,6 \\
\hline & & $\mathrm{Dp}$ & 11,8 & 14,6 & 17,1 & 15,9 & 15,9 & 13,9 & 18,8 & 15,7 \\
\hline & & P-Valor & $0,007^{*}$ & $0,038^{*}$ & $0,000^{*}$ & $0,04 *$ & $0,000 *$ & $0,006^{*}$ & $0,031 *$ & $0,000 *$ \\
\hline \multirow{7}{*}{$\begin{array}{l}\text { Hipertensão } \\
\text { arterial }\end{array}$} & Não & $\mathrm{N}$ & 91 & 91 & 91 & 91 & 91 & 91 & 91 & 91 \\
\hline & & Média & 84,3 & 85,6 & 65,3 & 74,1 & 66,2 & 78,7 & 85,2 & 69,9 \\
\hline & & $\mathrm{Dp}$ & 15,7 & 27 & 20,9 & 17,3 & 19,3 & 22,1 & 30 & 18,5 \\
\hline & Sim & $\mathrm{N}$ & 19 & 19 & 19 & 19 & 19 & 19 & 19 & 19 \\
\hline & & Média & 80,3 & 80,3 & 60,5 & 61,7 & 65,3 & 81,6 & 89,5 & 74,1 \\
\hline & & $\mathrm{Dp}$ & 12,4 & 25,8 & 17,7 & 14,2 & 16,4 & 18,8 & 19,4 & 18,7 \\
\hline & & P-Valor & 0,291 & 0,436 & 0,36 & $0,005^{*}$ & 0,85 & 0,605 & 0,553 & 0,373 \\
\hline \multirow{7}{*}{ Obesidade } & Não & $\mathrm{N}$ & 81 & 81 & 81 & 81 & 81 & 81 & 81 & 81 \\
\hline & & Média & 86,60 & 86,73 & 66,48 & 74,25 & 67,84 & 80,25 & 87,65 & 71,16 \\
\hline & & Dp & 14,23 & 25,34 & 20,57 & 16,77 & 18,37 & 20,72 & 27,10 & 19,47 \\
\hline & $\overline{S i m}$ & $\mathrm{~N}$ & 29 & 29 & 29 & 29 & 29 & 29 & 29 & 29 \\
\hline & & Média & 74,66 & 77,59 & 58,31 & 65,00 & 60,34 & 75,43 & 81,61 & 68,00 \\
\hline & & $\mathrm{Dp}$ & 14,70 & 30,14 & 18,82 & 17,87 & 19,04 & 23,97 & 31,61 & 16,63 \\
\hline & & P-Valor & $0,000^{*}$ & 0,116 & 0,063 & $0,014^{*}$ & 0,065 & 0,305 & 0,326 & 0,438 \\
\hline \multirow{7}{*}{ Depressão } & Não & $\mathrm{N}$ & 91 & 91 & 91 & 91 & 91 & 91 & 91 & 91 \\
\hline & & Média & 84,84 & 86,81 & 66,25 & 72,74 & 67,31 & 80,63 & 88,28 & 72,70 \\
\hline & & Dp & 14,95 & 24,53 & 20,05 & 17,70 & 18,76 & 19,21 & 26,00 & 17,93 \\
\hline & Sim & $\mathrm{N}$ & 19 & 19 & 19 & 19 & 19 & 19 & 19 & 19 \\
\hline & & Média & 76,84 & 72,37 & 55,11 & 67,37 & 58,95 & 71,05 & 75,44 & 58,95 \\
\hline & & Dp & 15,20 & 34,25 & 19,81 & 16,02 & 17,61 & 30,06 & 36,59 & 18,80 \\
\hline & & P-Valor & $0,037 *$ & $0,032 *$ & $0,029 *$ & 0,225 & 0,077 & 0,079 & 0,072 & $0,003 *$ \\
\hline
\end{tabular}


Tabela 4 - Correlação entre os domínios do questionário SF-36 com sexo e condições crônicas de saúde autorreferidas em servidores de uma universidade pública, na cidade de Cascavel

\begin{tabular}{|c|c|c|c|c|c|c|c|c|c|c|}
\hline & & & & & & & & & & conclusão \\
\hline \multirow{7}{*}{$\begin{array}{l}\text { Doença } \\
\text { respiratória }\end{array}$} & Não & $\mathrm{N}$ & 89 & 89 & 89 & 89 & 89 & 89 & 89 & 89 \\
\hline & & Média & 84,21 & 85,39 & 65,36 & 72,52 & 66,85 & 80,62 & 91,01 & 70,52 \\
\hline & & $\mathrm{Dp}$ & 15,09 & 25,51 & 20,70 & 17,66 & 18,61 & 20,56 & 22,34 & 18,80 \\
\hline & Sim & $\mathrm{N}$ & 21 & 21 & 21 & 21 & 21 & 21 & 21 & 21 \\
\hline & & Média & 80,24 & 79,76 & 59,95 & 68,81 & 61,67 & 72,02 & 65,08 & 69,52 \\
\hline & & $\mathrm{Dp}$ & 15,77 & 32,23 & 18,71 & 16,71 & 19,26 & 24,97 & 40,11 & 18,91 \\
\hline & & P-Valor & 0,284 & 0,39 & 0,276 & 0,384 & 0,256 & 0,101 & $0,000 *$ & 0,828 \\
\hline \multirow{7}{*}{ Artrite reumatoide } & Não & $\mathrm{N}$ & 103 & 103 & 103 & 103 & 103 & 103 & 103 & 103 \\
\hline & & Média & 84,27 & 84,47 & 64,98 & 72,72 & 66,41 & 78,40 & 85,76 & 71,77 \\
\hline & & Dp & 14,83 & 26,69 & 20,24 & 17,44 & 19,04 & 21,88 & 29,00 & 18,04 \\
\hline & Sim & $\mathrm{N}$ & 7 & 7 & 7 & 7 & 7 & 7 & 7 & 7 \\
\hline & & Média & 71,43 & 82,14 & 54,71 & 58,43 & 57,86 & 87,50 & 90,48 & 49,14 \\
\hline & & $\mathrm{Dp}$ & 17,25 & 31,34 & 21,31 & 12,15 & 12,20 & 16,14 & 16,27 & 17,08 \\
\hline & & P-Valor & $0,030 *$ & 0,826 & 0,198 & $0,036^{*}$ & 0,246 & 0,283 & 0,672 & $0,002 *$ \\
\hline \multirow{7}{*}{ DORT } & Não & $\mathrm{N}$ & 87 & 87 & 87 & 87 & 87 & 87 & 87 & 87 \\
\hline & & Média & 84,54 & 85,92 & 66,48 & 73,62 & 66,55 & 80,60 & 89,27 & 71,91 \\
\hline & & $\mathrm{Dp}$ & 15,45 & 25,77 & 20,04 & 17,82 & 18,70 & 22,13 & 26,16 & 18,19 \\
\hline & Sim & $\mathrm{N}$ & 23 & 23 & 23 & 23 & 23 & 23 & 23 & 23 \\
\hline & & Média & 79,35 & 78,26 & 56,17 & 64,96 & 63,26 & 72,83 & 73,91 & 64,35 \\
\hline & & $\mathrm{Dp}$ & 13,92 & 30,44 & 19,91 & 14,42 & 19,16 & 18,71 & 33,27 & 20,00 \\
\hline & & P-Valor & 0,147 & 0,225 & $0,030 *$ & $0,034 *$ & 0,457 & 0,125 & $0,020 *$ & 0,085 \\
\hline \multirow{7}{*}{$\begin{array}{l}\text { Gastrite/ } \\
\text { Úlcera }\end{array}$} & Não & $\mathrm{N}$ & 83 & 83 & 83 & 83 & 83 & 83 & 83 & 83 \\
\hline & & Média & 84,94 & 86,75 & 67,58 & 74,35 & 67,83 & 81,63 & 88,76 & 72,72 \\
\hline & & Dp & 15,09 & 25,10 & 20,52 & 15,92 & 18,68 & 19,94 & 25,65 & 17,77 \\
\hline & Sim & $\mathrm{N}$ & 27 & 27 & 27 & 27 & 27 & 27 & 27 & 27 \\
\hline & & Média & 78,89 & 76,85 & 54,33 & 64,00 & 59,81 & 70,83 & 77,78 & 62,96 \\
\hline & & $\mathrm{Dp}$ & 15,02 & 30,95 & 16,51 & 19,90 & 18,00 & 24,76 & 34,59 & 20,05 \\
\hline & & P-Valor & 0,073 & 0,096 & $0,003 *$ & $0,007 *$ & 0,053 & $0,023 *$ & 0,08 & $0,018 *$ \\
\hline
\end{tabular}

Obs: DORT: Doença Osteomuscular Relacionada com o Trabalho; CF: Capacidade Funcional; LAF: Limitações por aspectos físicos; EGS: Estado geral de saúde; Vit.: Vitalidade; AS: Aspectos sociais; LAE: Limitações por aspectos emocionais; SM: Saúde mental. Teste T-Student para a comparação de médias ao nível de 5,0\%. Fonte: Autoria própria (2012).

Quando relacionados os domínios do SF-36 com o sexo (Tabela 4), percebe-se que todos os domínios foram significativos $(\mathrm{p}<0,05)$. O sexo masculino apresentou as maiores pontuações em todos os domínios quando comparados ao sexo feminino. Isso pode indicar que os homens apresentam melhores percepções de QVRS quando comparados as mulheres. Estes achados corroboram com outros estudos (YOUSSEF; MOUBARAK; KAMEL, 2005, PAPP et al., 2011). De acordo com Youssef, Moubarak e Kamel (2005) é possível que os valores mais altos dos domínios para o sexo masculino sejam decorrentes da capacidade do homem em tolerar melhor as doenças, sem se afetar emocionalmente e tão intensamente quanto as mulheres. Este resultado pode, ainda, estar associado à maior frequência com que as mulheres relatam insatisfações e frustrações pessoais (CARVALHO et al., 2012). 
Quando se correlaciona os domínios do SF-36 com servidores que referem Hipertensão arterial (Tabela 4), apenas para o Estado geral de saúde $(61,7 \pm 18,37)$ demonstrou resultados significativos $(\mathrm{p}<0,05)$. Os valores dos domínios reduzidos para os servidores hipertensos podem estar relacionados à própria etiologia da doença e ao tratamento desta. Acredita-se que a ideia de gravidade da doença do ponto de vista do paciente tenha grande influência no resultado desse domínio. Este resultado corrobora com o encontrado em estudos realizados por Cavalcante et al. (2007) e Carvalho et al. (2012).

Para a correlação entre os domínios do SF-36 e Obesidade (Tabela 4), observou-se que os servidores com obesidade obtiveram valores menores para todos os domínios de QVRS do que os indivíduos com peso normal ou sobrepeso, sendo os resultados significativos para Capacidade funcional $(74,66 \pm 14,70)$ e Estado geral de saúde $(65,0 \pm 17,87)$. Assim, pode-se dizer que a obesidade está diretamente relacionada com a percepção e a redução das pontuações dos domínios acima citados, o que corrobora como os achados por outros autores (FLETCHER et al., 1998; KARINI; CUNHA, 2011). A relação direta entre a obesidade e a QV está intimamente ligada ao representativo excesso de peso, mais tendência ao isolamento social, estresse, depressão e agravamento da Capacidade funcional (TAVARES; NUNES; SANTOS, 2010).

Quando correlacionados Saúde mental com Depressão (Tabela 3), observou-se que os valores foram significativos $(\mathrm{p}<0,05)$ para este domínio e para Capacidade funcional $(76,84)$, Limitações por aspectos físicos $(72,37)$ e Dor $(55,11)$. Entretanto, todos os domínios apresentaram escores menores do que quando analisados para servidores sem manifestações de depressão. Em estudo com indivíduos portadores de hipertensão arterial utilizando outros instrumentos de mensuração de QVRS (WHOQLBref e MINICHAL-Br) foi observado que a depressão contribuiu para resultados reduzidos para todos os domínios avaliados (CARVALHO et al., 2012). Os achados do presente estudo corroboram com Carvalho et al. (2012) e Ware Jr. e Sherbourne (1992). Considera-se que a depressão diminua, não apenas a autoestima do indivíduo, mas, também, sua própria percepção da vida, da saúde e da doença, observando-se menor interesse na realização das atividades diárias do que para indivíduos não portadores da doença.

Quando analisados os domínios com as Doenças respiratórias (Tabela 4), o domínio Limitação por aspecto psicológico apresentou resultado significativo, com $\mathrm{p}=0,000$, na comparação entre servidores não portadores $(91,01 \pm 22,34)$ e portadores de patologia respiratória crônica $(65,08 \pm 40,11)$. Um estudo observou que portadores de doenças respiratórias crônicas são mais susceptíveis as mudanças no humor, como depressão e ansiedade, decorrente de autopercepção negativa e diminuição de capacidade física (RAMOS-CERQUEIRA; CREPALDI, 2000). Estes achados podem justificar o resultado significativo encontrado no domínio analisado no presente trabalho.

Em relação aos servidores portadores de Artrite, os domínios que apresentaram significância foram Capacidade funcional $(\mathrm{p}=0,03)$, Estado geral de saúde $(\mathrm{p}=0,036)$ e Saúde mental $(\mathrm{p}=0,002)$, demonstrando pior percepção de QV pelos servidores. Corbacho e Dapueto (2010) em estudo de QV em indivíduos portadores de artrite discorrem que fatores envolvidos na percepção de dor e de saúde refletem diretamente na percepção de $\mathrm{QV}$, entre estas, dificuldade de tratamento, dores e fraquezas frequentes.

Na correlação dos domínios com DORT, os domínios Dor $(56,17 \pm 19,91)$, Estado geral de saúde $(64,96 \pm 14,42)$ e Limitações por aspectos emocionais $(73,91 \pm 33,27)$ apresentaram significância estatística. Helfenstein Jr. e Feldman (1998) também observaram que indivíduos com DORT apresentam altos índices de ansiedade e depressão, o que pode levar a alteração da percepção da dor e outros sintomas orgânicos. Em estudo de Capela et al. (2009), indivíduos com dor difusa e crônica apresentaram sintomas mais intensos de depressão, ansiedade e pior QV.

Quando investigada a correlação entre os domínios e Gastrite/Úlcera, os resultados demonstraram significância para os domínios Dor $(54,33 \pm 16,51)$, Estado geral de saúde $(64,00 \pm 19,90)$, Aspectos Sociais $(70,83 \pm 24,76)$ e Saúde Mental $(62,96 \pm 20,05)$. Considerando a gastrite uma doença psicossomática, pode-se compreender que indivíduos estressados poderão apresentar alterações em seus aspectos psicológicos e de percepção de doença (BRANDAO; FERRAZ; ZERBINI, 1997). Portanto, é possível que a redução dos escores dos domínios Estado geral de saúde, Aspectos Sociais, Saúde mental e Dor, estejam mais ligadas ao fator psicológico de estresse, do que a patologia gastrite. 
A QV só pode ser avaliada pela percepção do próprio indíviduo e, assim, os instrumentos de avaliação devem contemplar a perspectiva do indíviduo e ser composto por diferentes dimensões, dentro dos componentes de saúde física e mental. Analisar a QV sob a pespectiva de sua relação com a saúde implica na inclusão de aspectos associados à enfermidades e à intervenções de saúde (SEIDL; ZANNON, 2014). O instrumento SF 36 apresenta-se como um bom instrumento para se avaliar a QVRS por ser capaz de detectar varias dimensões da QVRS comprometidas.

Ressalta-se a importância de conhecer as doenças prevalentes entre servidores públicos para se determinar demandas de ações de prevenção e de melhoria de QV, uma vez que escores de domínios mais baixos foram observados com maior frequência entre os servidores portadores de doenças crônicas.

\section{Considerações finais}

Na presente pesquisa à correlação do SF-36 com o sexo demonstrou que todos os domínios tiveram escores maiores para o sexo masculino, traduzindo-se em uma melhor QVRS nos homens. Corroborando com a literatura, onde as mulheres queixam-se mais e os homens tendem a tolerar mais as adversidades.

O domínio Dor foi o mais comprometido na amostra estudada. Este resultado foi associado à prevalência de DORT e outras doenças ou dores musculares, as quais são muito frequentes em servidores públicos. Estas doenças são responsáveis por gerar demasiada dor, podendo levar o indivíduo a incapacitação funcional.

Embora se constate na literatura estudos que investigam a QVRS em trabalhadores, nos servidores público ainda é pouco investigada. A presente pesquisa permitiu conhecer a QVRS em servidores públicos e a correlação com as condições crônicas de saúde, demonstrando em geral altas médias para os domínios de QVRS. No entanto, os escores mais afetados podem ser melhorados.

\section{Referências}

BARRETO, J. Envelhecimento e qualidade de vida: o desafio actual. Revista Faculdade de Letras: Sociologia, v. 15, n. 1, p. 289-302, 2005.

BERLIM, M. T.; FLECK, M. P. A. Quality of life: a brand new concept for research and practice in psychiatry. Revista Brasileira de Psiquiatria, São Paulo, v. 25, n. 4, p. 249-252, out. 2003. Crossef

BRANDÃO, L.; FERRAZ, M. B.; ZERBINI, C. A. F. Avaliação da qualidade de vida na artrite reumatoide: revisão atualizada. Revista Brasileira de Reumatologia, v. 37, n. 5, set./out. 1997.

CAPELA, C.; MARQUES, A. P.; ASSUMPÇÃO, A.; SAUER, J. F.; CAVALCANTE, A. B.; CHALOT, S. D. Associação da qualidade de vida com dor, ansiedade e depressão. Fisioterapia e Pesquisa, São Paulo, v. 16, n. 3, p. 263-268, jul./set. 2009. Crossef

CARVAlHO, M. A. N.; SILVA, I. B. S.; RAMOS, S. B. P.; COELHO, L. F.; GONÇALVES, I. D.; FIGUEIREDO NETO, J. A qualidade de vida de pacientes hipertensos e comparação entre dois instrumentos de medida de QVRS. Arquivos Brasileiros de Cardiologia, v. 98, n. 5, p. 442-451, 2012. erossef

CAVAlCANTE, M. A.; BOMBIG, M. T. N.; LUNA FILHO, B.; CARVAlHO, A. C. C.; PAOLA, A. A. V.; PÓVOA, R. Qualidade de vida de pacientes hipertensos em tratamento ambulatorial. Arquivos Brasileiros de Cardiologia, v. 89, p. 245-250, 2007. rossef

CICONELLI, R. M.; FERRAZ, M. B.; SANTOS, W.; MEINÃO, I.; QUARESMA, M. R. Tradução para a língua portuguesa e validação do questionário genérico de avaliação de qualidade de vida SF-36 (Brasil SF-36). Revista Brasileira de Reumatologia, v. 39, n. 3, p. 153-150, maio/jun. 1999. 
CORBACHO, M. I.; DAPUETO, J. J. Avaliação da capacidade funcional e da qualidade de vida de pacientes com artrite reumatoide. Revista Brasileira de Reumatologia, v. 50, n. 1, p. 31-43, 2010. crossef

FARHAT, F. G.; ZAZINI, A. C. Qualidade de vida relacionada à saúde - QVRS. Revista Racine, v. 47, n. 4, p. 11-19, nov./dez. 1998.

FLETCHER, A. E.; BULPITT, C. J.; TUOMILEHTO, J.; BROWNE, J.; BOSSINI, A.; KAWCKAJASZCZ, K.; KIVINEN, P.; O’BRIEN, E.; STAESSEN, J.; THIJS, L.; VANSKA, O.; VANHANEN, H. Quality of life of elderly patients with isolated systolic hypertension: baseline data from Syst-Eur trial. Journal of Hypertension, v. 16, n. 8, p. 1117-1124, Aug. 1998. rossef

HELFENSTEIN JR., M.; FELDMAN, D. Prevalência da síndrome da fibromialgia em pacientes diagnosticados como portadores de lesões por esforções repetitivos (LER). Revista Brasileira de Reumatologia, v. 36, n. 2, mar./abr. 1998.

KARINI, G. O.; CUNHA, G. B. O impacto da obesidade e do sobrepeso nos níveis de qualidade de vida percebida em homens e mulheres da cidade de Aveiro, Portugal. EFDeportes.com, Revista Digital, $n$. 156, ano. 16, maio 2011.

MARTINS, L. M.; FRANÇA, A. P. D.; KIMURA, M. Qualidade de vida de pessoas com doença crônica. Revista Latino-americana de Enfermagem, Ribeirão Preto, v. 4, n. 3, p. 5-18, dez. 1996.

crossef

MARTINS, M. R. I.; CESARINO, C. B. Qualidade de vida de pessoas com doença renal crônica em tratamento hemodialítico. Revista Latino-Americana de Enfermagem, v. 13, n. 5, p. 670-676, set./out. 2005. rossef

MENDES, R. Impacto dos efeitos da ocupação sobre a saúde dos trabalhadores. Revista de Saúde Pública, São Paulo, v. 22, n. 4, p. 311-326, 1988. Crossef

PAPP, M. R.; SOUZA, R. C.; LIMA, S. M. P. F.; MATSUMOTO, M. H.; CHAMLIAM, T. R; SANTOS, J. B. G. Comparação entre DASH e SF-36 do cotovelo traumatizado reabilitado na terapia ocupacional. Acta Ortopédica Brasileira, v. 19, n. 6, p. 356-361, 2011. rossef

PIMENTA, F. A. P.; SIMIL, F. F.; TORRES, H. O. G.; AMARAL, C. F. S.; REZENDE, C. F.; COELHO, T. O.; REZENDE, N. A. Avaliação da qualidade de vida de aposentados com a utilização do questionário SF-36. Revista da Associação Médica Brasileira, v. 54, n. 1, p. 55-60, 2008. rossef

PINHEIRO, S. S.; SUGAHARA, T. Perfil dos funcionários públicos ativos nas áreas federal, estadual e municipal - comparação de bases disponíveis: RAIS, PNAD e SIAPE. Texto para Discussão (IPEA. Brasília), Rio de Janeiro, n. 837, out. 2001.

PROBST, E. R.; RAMOS, P. A evolução da mulher no mercado de trabalho. Revista Leonardo Pós Órgão de Divulgação Cientifica e Cultura do Instituto Catarinense de Pós-Graduação, v. 2, n. 1, p. 1-8, jan./jun. 2003.

RAMOS-CERQUEIRA, A. T. A.; CREPALDI, A. L. Qualidade de vida em doenças pulmonares crônicas: aspectos conceituais e metodológicos. Jornal de Pneumologia, v. 26, n. 4, p. 207-213, jul./ago. 2000. 
SCHUlZ, R. B.; ROSSIGNOLI, P.; CORRER, C. J.; FERNÁNDEZ-LliMÓS, F.; TONI, P. M. Validação do Mini-Questionário de Qualidade de Vida em Hipertensão Arterial (MINICHAL) para o Português (Brasil). Arquivos Brasileiros de Cardiologia, São Paulo, v. 90, n. 2, p. 139-144, fev. 2008. rossef

SEIDL, E. M. F.; ZANON, C. M. L. C. Qualidade de vida e saúde: aspectos conceituais e metodológicos. Cadernos de Saúde Pública, Rio de Janeiro, v. 20, n. 2, p. 580-588, mar./abr. 2004.

SILQUEIRA, S. M. F. O questionário genérico SF-36 como instrumento de mensuração da qualidade de vida relacionada a saúde de pacientes hipertensos. 2005. 112 p. Tese (Doutorado em Enfermagem em Saúde Pública) - Programa de Pós-Graduação em Enfermagem em Saúde da Escola de Enfermagem, Universidade de São Paulo, Ribeirão Preto, 2005.

TAVARES, T. B.; NUNES, S. M.; SANTOS, M. O. Obesidade e qualidade de vida: revisão da literatura. Revista de Medicina de Minas Gerais, v. 20, n. 3, p. 359-366, 2010.

URZUA, M. A. Calidad de vida relacionada con la salud: elementos conceptuales. Revista Médica del Chile, Santiago, v. 138, n. 3, mar. 2010.

WARE JR, J. E. SF-36 health survey update. Spine (Phila Pa 1976), v. 25, n. 24, p. 3130-3139, 2000. erossef

WARE JR., J. E.; SHERBOURNE, C. D. The MOS 36-Item Short-Form Health Survey (SF-36). Medical Care, v. 30, n. 6, p. 473-481, June 1992.

WORLD HEALTH ORGANIZATION. Global database on body mass index: BMI classification. Secondary Global database on body mass index: BMI classification, 2008. Disponível em: <http://apps.who.int/bmi/index.jsp?introPage=intro_3.html>. Acesso em: 19 jan. 2015.

YOUSSEF, R. M.; MOUBARAK, I. I.; KAMEL, M. I. Factors affecting the quality of life of hypertensive patients. Eastern Mediterranean Health Journal, v. 11, n. 1-2, p. 109-118, 2005.

ZORTEA, K. Qualidade de vida em doenças crônicas. Arquivos Brasileiros de Cardiologia, São Paulo, v. 94, n. 5, maio 2010. 